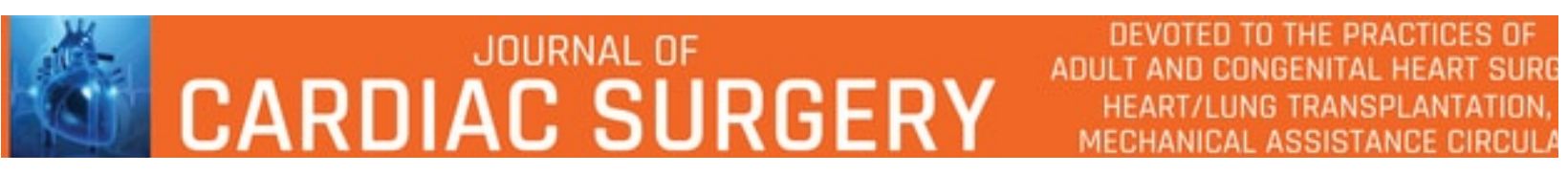

\title{
SUCCESSFUL LEFT VENTRICULAR ASSIST DEVICE MANAGEMENT REQUIRES MORE THAN JUST A PRIME PUMP
}

\begin{tabular}{|r|l|}
\hline Journal: & Journal of Cardiac Surgery \\
\hline Manuscript ID & JOCS-2021-IC-104 \\
\hline Manuscript Type: & Invited Commentary \\
\hline Author: & 17-Jan-2021 \\
\hline Complete List of Authors: & $\begin{array}{l}\text { Brahmbhatt, Darshan; Toronto General Hospital, Cardiology and Heart } \\
\text { Transplant } \\
\text { Billia, Filio; Toronto General Hospital, Cardiology and Heart Transplant } \\
\text { Rodger, Marnie; Peter Munk Cardiac Centre, } \\
\text { Rao, Vivek; Toronto General Hospital, Cardiology and Heart Transplant }\end{array}$ \\
\hline Keywords: & mechanical circulatory support, heart failure \\
\hline &
\end{tabular}

\section{SCHOLARONE ${ }^{m}$ \\ Manuscripts}




\title{
SUCCESSFUL LEFT VENTRICULAR ASSIST DEVICE MANAGEMENT REQUIRES MORE THAN JUST A PRIME PUMP
}

\author{
Authors: \\ Darshan H. Brahmbhatt MA, MRCS, MRCP1,2,3 (Darshan.Brahmbhatt@uhn.ca, ORCID: 0000-0003-3440- \\ 0523) \\ Filio Billia MD, PhD ${ }^{1,3}$ (Phyllis.Billia@uhn.ca, ORCID: 0000-0002-2824-1215) \\ Marnie Rodger, ACNP, $\mathrm{RN}^{1,3,4}$ \\ Vivek Rao MD, PhD, FRCSC, FAHA ${ }^{3,4 *}$ (Vivek.Rao@uhn.ca, ORCID: 0000-0003-1920-0937)
}

\section{Affiliations:}

1. Peter Munk Cardiac Centre, Division of Cardiology, University Health Network, University of Toronto, Toronto, Canada

2. National Heart \& Lung Institute, Imperial College London, London, United Kingdom

3. Ted Rogers Centre for Heart Research, Toronto, Canada

4. Peter Munk Cardiac Centre, Division of Cardiovascular Surgery, University Health Network, University of Toronto, Toronto, Canada

*Corresponding author

Dr. Vivek Rao

University Health Network

$4 \mathrm{~N} 457$

200 Elizabeth Street

Toronto, Ontario

M5G 2C4

Tel: +1 416 340-3562

Fax: +1416 340-3337

Email:Vivek.Rao@uhn.ca

Total word count: 1,321 without references 


\section{Acknowledgements}

All authors contributed to the conceptualization and writing of the manuscript.

\section{Conflicts of Interest}

DHB has received travel support from Abbott \& Biotronik, and honoraria, travel support and research funding from Boston Scientific. VR has received consultancy fees from Abbott and Medtronic.

\section{Funding}

None 
The evolution in mechanical circulatory support devices has occurred alongside advances in cardiac transplantation. The lack of availability of suitable organs for transplantation while patients were admitted with deteriorating advanced heart failure state led to the development of temporary ventricular assist devices that demonstrated superior outcomes compared to optimal medical management (1). These paracorporeal devices required patients to remain in hospital waiting for a suitable donor organ to become available. Over time, these devices have become more durable and subsequent generations of left ventricular assist devices (LVAD) have become smaller and the pump entirely contained within the body, allowing ambulatory circulatory support. The role of LVADs has also evolved, with devices now implanted not only as a bridge to transplantation, but also as destination therapy (DT) in those patients who are ineligible for transplant, as a long-term solution for their symptomatic end-stage advanced heart failure.

The greatest challenge to LVAD use, particularly as DT, has been the significant burden of complications and re-admission rate. This has an effect on both the quality of life of the patient and the cost-benefit of such interventions. Our program has previously reported complication rates for durable continuous-flow LVAD, with $63 \%$ of patients requiring re-admission after device implantation, at a rate of 2.23 events per patient-year. The commonest cause for re-admission was infection (17\%), followed by LVAD malfunction (14\%), neurological (12\%), and gastrointestinal bleeding $(9 \%)(2)$. As the technology has become more reliable, DT LVAD implantation has become reliable. A move to using centrifugal-flow pumps rather than axial-flow pumps has reduced the hemocompatibility issues associated with LVADs, such as thrombus formation, stroke and bleeding exacerbated by fragmentation of von Willebrand factor(3). Newer devices, such as the centrifugal-flow LVAD designs of the HeartWare (Medtronic, Minneapolis, MN) and HeartMate 3 (Abbott, Abbott Park, IL), have seen fewer mechanical complications with the device itself. The MOMENTUM 3 trial compared the fully magnetically levitated centrifugal-flow HeartMate 3 LVAD against the axial-flow Heartmate II device (Abbott, Abbott Park, IL). After two years follow-up, 
significantly more patients with the HeartMate 3 device were alive and free of disabling stroke or requiring reoperation to replace or remove a malfunctioning device (relative risk 0.84 ; $95 \%$ confidence interval $[\mathrm{Cl}]$ 0.78 to $0.91 ; \mathrm{P}<0.001)(4)$. There were also fewer hospitalisations in the HeartMate 3 patient group (2.26 vs 2.47 events per patient-year, hazard ratio $0.92 ; 95 \% \mathrm{Cl} 0.86-0.99)$ with admissions significantly shorter ( 5 days shorter; $95 \% \mathrm{Cl} 1.3$ to 8.7 ). Nevertheless, these newer devices have not reduced the rates of device infection or right heart failure associated with LVAD therapy(4).

Within this context of ongoing risk of LVAD complication and need for re-admission after implantation, the report by Stonebraker and colleagues of a patient who has avoided unplanned hospitalisation for over four years is remarkable, and the team at University of Chicago should be congratulated for this achievement. However, this accomplishment needs to be placed into the context of the durable mechanical circulatory program at their institution. It is unclear if this is a singular occurrence of admission-avoidance, or whether the clinic itself has developed strategies to reduce the complications associated with LVAD therapy. The authors report on use of beta-blocker medication and omega-3 to reduce heart failure and gastrointestinal bleeding complications, respectively. The use of beta-blockers is commonplace in heart failure management, so it is unclear how this intervention has resulted in a lack of hospitalisation, and there are no prospective, randomised controlled trials evidencing the benefit of omega-3 in LVAD patients. Without the context of the how the LVAD program is managed at their institution, one could ungenerously ascribe this report to good fortune rather than the expert management of LVAD patients which is more likely.

Since 2006 at our own institution, a total of 76 patients have been supported by durable continuous-flow LVAD for greater than one year, with $9(12 \%)$ re-admitted for heart failure decompensation within the first year after implantation. From within this cohort, a 43-year-old male patient who had a HeartMate 3 LVAD implanted in 2014, initially as bridge to heart transplant candidacy for idiopathic dilated cardiomyopathy, has been supported for over 2,250 days (greater than 6 years) without clinical 
deterioration requiring hospitalisation. He was discharged 21 days after LVAD implantation, and has subsequently thrived with his device, to the extent that he requested to be removed from the transplant waiting list as he was doing so well. We believe that his successful post-operative course is due to the management protocols in place in our LVAD clinic.

We have previously published our institutional schedules for monitoring and follow-up of patients implanted with a durable LVAD and how this has had to be altered due to the SARS-CoV-2 pandemic(5). We are now more reliant on remote patient monitoring to avoid hospital-contact for our patients who are at higher risk of complications from the novel coronavirus. Decisions to implement changes such as these are taken at a programmatic level and add to the combination of device-related, patient-related and clinicrelated factors that determine patient outcomes after LVAD implantation (figure 1). Given that DT LVAD therapy now accounts for the majority of durable devices in the USA, with the latest annual report of the Society of Thoracic Surgeons Intermacs Registry reporting over $70 \%$ of the 1752 implants from January to September 2019 implanted for this reason, patients will be supported for longer durations on LVAD therapy(6).

As the devices become able to support patients for longer periods of time, the clinical management of the LVAD patient needs to be better designed, with more-evidence based guidelines developed for LVAD programs. Optimal management of anticoagulation remains unclear, especially in those patients who have had previous bleeding events. Some evidence suggests running patients at lower levels of anticoagulation than the LVAD product guide recommends may be safe and reduce bleeding events, while other groups have focussed on increasing time in therapeutic anticoagulant range as a means to reduce thrombotic events $(7,8)$. Infection management has not changed much since the first generation of LVADs and most institutions use their own protocols for treatment of device or driveline infections, often heavily dependent on surgeon and centre preference and experience. More recently networks have been established to ensure a rational approach to infection prevention and management in these patients with 
audit of outcomes aiming to lead to improvement in care(9). Finally, avoiding heart failure hospitalisation remains a challenge. The monitoring of haemodynamic parameters through telehealth has been rudimentary for LVAD patients, with patients or their caregivers having to telephone the LVAD clinic to provide device parameters such as speed, calculated flow and power consumption. This is the case despite the two largest LVAD manufacturers having well-established remote monitoring networks for pacemaker and ICD management (Medtronic Carelink ${ }^{\mathrm{TM}}$ and Abbott merlin.net ${ }^{\mathrm{TM}}$ ) but no means to transmit LVAD information electronically to healthcare providers. There is some promise in guiding LVAD settings through continuous non-invasive haemodynamic measurement with CardioMEMS (Abbott, Abbott Park, IL) pulmonary artery pressure monitoring, though we remain some way away from closed loop LVAD speed optimisation guided by implanted haemodynamic monitors(10). Until then, in-person ramp studies, guided by echocardiography or invasive haemodynamic testing, remain the only means of LVAD optimisation.

LVAD therapy is mandated by numerous guidelines in the management of patients with advanced heart failure who require long-term circulatory support either as a bridge to cardiac transplantation or as destination therapy in those deemed transplant-ineligible(11-13). Despite advances in technology and device performance, complication and readmission rates remain high, carrying with them significant cost implications that may act as a barrier to patients receiving LVAD therapy(14). While it is important to celebrate successes in avoiding hospitalisation for four years, or even six years, one swallow does not a summer make. To ensure that the largest number of heart failure patients benefit from this treatment, LVAD programs need to develop new paradigms of care buttressed by evidence of clinical effectiveness to generate sustained improvement in the care and associated outcomes of all VAD patients.

Figure 1: Determinants of clinical outcomes in LVAD patients. This figure has been designed using resources from Freepik.com 


\section{References}

1. Rose EA, Gelijns AC, Moskowitz AJ, Heitjan DF, Stevenson LW, Dembitsky W, et al. Long-term use of a left ventricular assist device for end-stage heart failure. N Engl J Med. 2001;345(20):1435-43. Epub 2002/01/17. doi: 10.1056/NEJMoa012175. PubMed PMID: 11794191.

2. Da Silva $M$, Maclver J, Rodger $M$, Jaffer $M$, Raju S, Billia $F$, et al. Readmissions Following Implantation of a Continuous-Flow Left Ventricular Assist Device. J Card Surg. 2016;31(5):361-4. Epub 2016/04/14. doi: 10.1111/jocs.12744. PubMed PMID: 27072942.

3. Proudfoot AG, Davidson SJ, Strueber M. von Willebrand factor disruption and continuous-flow circulatory devices. J Heart Lung Transplant. 2017;36(11):1155-63. Epub 2017/08/02. doi: 10.1016/j.healun.2017.06.004. PubMed PMID: 28756118.

4. Mehra MR, Uriel N, Naka Y, Cleveland JC, Jr., Yuzefpolskaya M, Salerno CT, et al. A Fully Magnetically Levitated Left Ventricular Assist Device - Final Report. N Engl J Med. 2019;380(17):1618-27. Epub 2019/03/19. doi: 10.1056/NEJMoa1900486. PubMed PMID: 30883052.

5. Amadio JM, Brahmbhatt DH, Ross HJ, Rao V, Billia F. The changing landscape of left ventricular assist device care in the setting of a pandemic. ESC Heart Fail. 2020;7(5):2140-2. Epub 2020/07/09. doi: 10.1002/ehf2.12845. PubMed PMID: 32638494; PubMed Central PMCID: PMCPMC7362049.

6. Teuteberg JJ, Cleveland JC, Jr., Cowger J, Higgins RS, Goldstein DJ, Keebler M, et al. The Society of Thoracic Surgeons Intermacs 2019 Annual Report: The Changing Landscape of Devices and Indications. Ann Thorac Surg. 2020;109(3):649-60. Epub 2020/03/03. doi: 10.1016/j.athoracsur.2019.12.005. PubMed PMID: 32115073.

7. Netuka I, Ivak P, Tucanova Z, Gregor S, Szarszoi O, Sood P, et al. Evaluation of low-intensity anticoagulation with a fully magnetically levitated centrifugal-flow circulatory pump-the MAGENTUM 1 study. 
J Heart Lung Transplant. 2018;37(5):579-86. Epub 2018/04/16. doi: 10.1016/j.healun.2018.03.002. PubMed PMID: 29655662.

8. Sage W, Gottiparthy A, Lincoln P, Tsui SSL, Pettit SJ. Improving anticoagulation of patients with an implantable left ventricular assist device. BMJ Open Qual. 2018;7(4):e000250. Epub 2018/10/12. doi: 10.1136/bmjoq-2017-000250. PubMed PMID: 30306143; PubMed Central PMCID: PMCPMC6173227.

9. Bernhardt AM, Schloglhofer T, Lauenroth V, Mueller F, Mueller M, Schoede A, et al. Prevention and early treatment of driveline infections in ventricular assist device patients - The DESTINE staging proposal and the first standard of care protocol. J Crit Care. 2020;56:106-12. Epub 2020/01/04. doi: 10.1016/j.jcrc.2019.12.014. PubMed PMID: 31896443.

10. Veenis JF, Brugts JJ. Pre-Operative Hemodynamic Guidance by Cardiomems to Improve LVAD Outcome. The Journal of Heart and Lung Transplantation. 2020;39(4):S446-S7. doi: 10.1016/j.healun.2020.01.265.

11. Potapov EV, Antonides C, Crespo-Leiro MG, Combes A, Farber G, Hannan MM, et al. 2019 EACTS Expert Consensus on long-term mechanical circulatory support. Eur J Cardiothorac Surg. 2019;56(2):23070. Epub 2019/05/18. doi: 10.1093/ejcts/ezz098. PubMed PMID: 31100109; PubMed Central PMCID: PMCPMC6640909.

12. National Institute of Health and Care Excellence (NICE). Implantation of a left ventricular assist device for destination therapy in people ineligible for heart transplantation: Interventional procedures guidance [IPG516]. NHS; 2015.

13. Feldman D, Pamboukian SV, Teuteberg JJ, Birks E, Lietz K, Moore SA, et al. The 2013 International Society for Heart and Lung Transplantation Guidelines for mechanical circulatory support: executive summary. J Heart Lung Transplant. 2013;32(2):157-87. Epub 2013/01/29. doi: 10.1016/j.healun.2012.09.013. PubMed PMID: 23352391. 
14. Baras Shreibati J, Goldhaber-Fiebert JD, Banerjee D, Owens DK, Hlatky MA. Cost-Effectiveness of Left Ventricular Assist Devices in Ambulatory Patients With Advanced Heart Failure. JACC Heart Fail. 2017;5(2):110-9. Epub 2016/12/27. doi: 10.1016/j.jchf.2016.09.008. PubMed PMID: 28017351. 


\section{DETERMINANTS OF OUTCOMES IN LVAD PATIENTS}

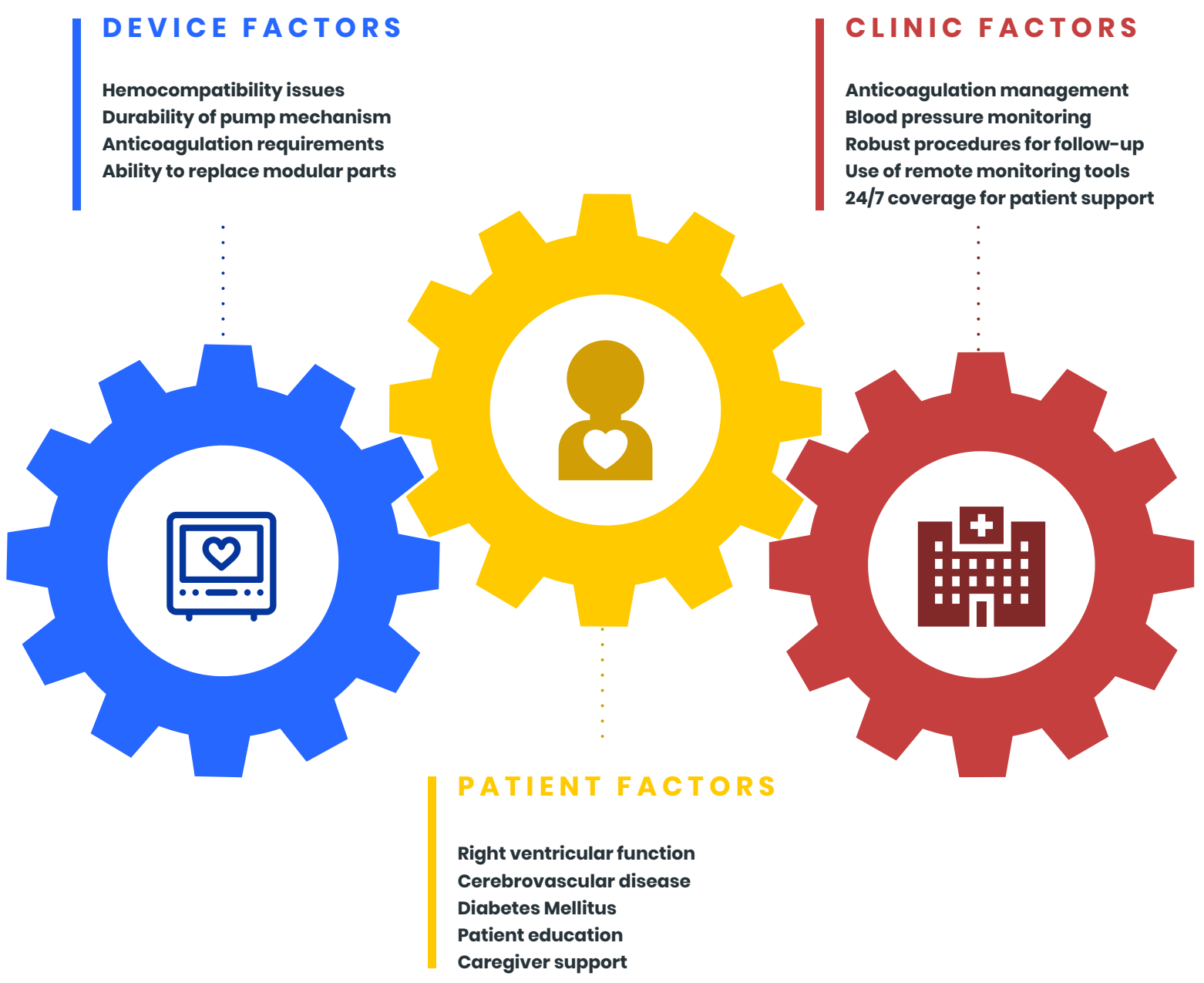

